\title{
Comparative Physicochemical and Biological Characterisation of the Similar Biological Medicinal Product Teriparatide and Its Reference Medicinal Product
}

\author{
Piroska Kovács ${ }^{1}$ - Tamás Schäfer ${ }^{1}$ - Viktor Háda ${ }^{1} \cdot$ Helga Hevér $^{1}$ - Sonja Klingelhöfer ${ }^{2} \cdot$ Merle Nebel $^{2} \cdot$ Tanja Stadie $^{2}$. \\ Róbert Kiss $^{1} \cdot$ Zoltán Urbányi $^{1}$ (1)
}

Published online: 8 October 2019

(c) The Author(s) 2019

\begin{abstract}
Background In January 2017, the European Commission approved Terrosa ${ }^{\circledR}$ (company code RGB-10) as one of the first biosimilar medicinal products of teriparatide for the same indications as for the reference medicinal product Forsteo ${ }^{\circledR}$ (Lilly France S.A.S.), which has been on the market in the European Union since 2003. The active pharmaceutical ingredient of the reference medicinal product is the biologically active 1-34 fragment of the endogenous human parathyroid hormone [PTH(1-34)]. It is one of the three bone anabolic agents used in the treatment of osteoporosis promoting bone formation and preventing fragility fractures.

Objective The objective of this paper is to summarise the results of the comparative analysis of representative batches of both the RGB-10 drug product and the reference medicinal product performed by physicochemical and in vitro biological methods. Methods A series of state-of-the-art analytical methods were applied in a comparative head-to-head manner for testing the similarity in respect to purity, content, structure and potency.

Results Based on the results of the comprehensive physicochemical and biological characterisation, RGB-10 proved to be highly similar to the reference medicinal product with respect to the critical quality attributes investigated.

Conclusion The results of the quality comparability study demonstrated similarity of RGB-10 to the reference medicinal product, providing the scientific basis for conducting a specifically designed clinical programme, and supported registration of the Marketing Authorisation Application of RGB-10 in the EU.
\end{abstract}

\section{Key Points}

RGB-10, a biosimilar of Eli Lilly’s Forsteo ${ }^{\circledR}$, has been approved by the European Commission for the treatment of osteoporosis.

The results of the quality comparability study proved that the representative batches of RGB-10 and the reference medicinal product are similar in the critical quality attributes, including purity, content of the active pharmaceutical ingredient and the excipients.

Zoltán Urbányi

z.urbanyi@richter.hu

1 Chemical Works of Gedeon Richter Plc, Gyömrôi út 19-21, Budapest 1103, Hungary

2 Richter Helm Biologics GmbH \& Co. KG, Suhrenkamp 59, 22335 Hamburg, Germany

\section{Introduction}

In January 2017, Terrosa ${ }^{\circledR}$ (RGB-10) was approved as one of the first biosimilar medicinal products of teriparatide by the European Commission (Marketing Authorisation Holder: Gedeon Richter Plc. [GR]). Teriparatide, produced by recombinant DNA technology, is a single-chain, 34-residue linear polypeptide without any post-translational modifications. The molecule shows complete sequence homology with the N-terminal 1-34 active fragment of the endogenous human parathyroid hormone (PTH), which promotes the remodelling of bone through binding and activation of the PTH-R1 receptor. It is one of the three currently available therapeutic products capable of promoting the formation of new bone tissue and improving bone quality [1]. The reference medicinal product (RMP) was approved by the US Food and Drug Administration (FDA) in November 2002 and by the EU Commission in June 2003 under the brand names of Forteo ${ }^{\circledR}$ and Forsteo ${ }^{\circledR}$ (Eli Lilly), respectively. 
The risk of developing osteoporosis becomes significant with age and the resultant fragility fractures, known as the main complications of the disease, often represent a lifechanging event in elderly patients due to the devastating impact on mortality, morbidity, and the quality of life [2]. The substantial increase in average life expectancy has brought about an osteoporosis epidemic, and as such an ever-increasing demand for effective preventive treatment options, rendering osteoporosis an enormous socioeconomic burden. Biopharmaceuticals represent a paradigm shift in the treatment of many disabling or life-threatening conditions and have revolutionised the management of some of the most difficult-to-treat diseases; however, due to being prohibitively expensive, many patients cannot benefit from them. Biosimilar medicinal products are biological products similar to the reference product in terms of structure, quality characteristics, biological activity, efficacy, safety and immunogenicity profile based on a comprehensive comparability exercise.

The comparability exercise, which is a specific, stepwise process for each product, involves the comprehensive head-to-head comparison of the biosimilar medicinal product and the reference medicinal product at quality, nonclinical and clinical levels. A robust quality comparability study, achieved through the application of orthogonal and state-of-the-art analytical techniques, is considered to be the most sensitive scientific indicator of similarity and should provide analytical confirmation that any differences observed in the quality attributes have no adverse impact on the safety or the efficacy of the medicinal product [3]. Therefore, the extent and nature of non-clinical and clinical studies depends on the level of evidence obtained in correct establishment of the analytical panel.

In the present paper, we summarise the results of the quality comparability study performed between RGB-10 and its European reference product, which provided strong basis for the clinical study and for the successful registration of RGB-10 in Europe [4].

\section{Materials and Methods}

\subsection{Tested Drug Products}

Twenty-seven RMP batches were analysed to establish a quality target product profile and comparability ranges in the frame of the project. Additionally, several RGB-10 batches produced in different scale were characterised, evaluating process improvement and biosimilarity during the whole development. Seven RGB-10 batches manufactured by the final commercial scale processes having equivalent quality were characterised to prove similar quality. Three batches of RGB-10 produced by the final commercial scale technology and three batches of RMP were selected and analysed in a head-to-head manner to conduct a comparability exercise for marketing authorisation. Here we compare the quality of one of each representative RGB-10 and RMP samples selected from the head-to-head comparability assessment. Their representativity of the samples was confirmed by their quality and by the manufacturing technology. Forsteo ${ }^{\circledR}$ batches used in comparability studies were sourced from the European market (Lilly France S.A.S, Fegersheim, France), supplied as the marketed pre-filled pen.

The drug substance (DS) of RGB-10 was manufactured by Richter-Helm Biologics, Bovenau, Germany. The active pharmaceutical ingredient (API) is expressed as a fusion protein in an Escherichia coli strain. After cleavage of the fusion protein, solubilisation and refolding, application of multiple orthogonal chromatography steps ensure the purity of the DS. RGB-10 drug product (DP) was manufactured by Gedeon Richter Plc. in Debrecen, Hungary. The RGB-10 DP has an identical formulation to that of the RMP, including metacresol as a preservative agent in aqueous buffer. Similarly to the RMP, RGB-10 is a multidose product administered as a daily $20 \mu \mathrm{g} / 80 \mu \mathrm{L}$ subcutaneous injection by means of a reusable pen injector.

\subsection{Methods Selection}

The analytical methods applied in the quality comparability study were selected to monitor all the critical quality attributes (CQAs) of the PTH(1-34)-containing DPs with capability of detecting even minor differences. In line with the relevant guidelines, the quality attributes of RGB-10 and their criticality were evaluated in the context of the product characteristics including the purity, the content of the active pharmaceutical ingredient and the excipients, molecular structure and potency [5]. Table 1 summarises the CQAs and the corresponding analytical methods applied.

Methods were developed not only to prove similarity but also to be capable of revealing even minor differences and were shown to be suitable for their intended purpose by qualification procedure. The analytical methods that are part of the specification together with the potency measurements went through a comprehensive validation procedure.

\subsection{Composition and Strength}

Reversed-phase high-performance liquid chromatography (RP-HPLC) experiments for quantitation of the API content were performed on a Shimadzu Nexera LC-30AD UHPLC system by using a C18 column $(100 \times 2.1 \mathrm{~mm}$, S-1.7 $\mu \mathrm{m}$ ) with gradient elution (Phase A: acetonitrile (ACN): water: trifluoroacetic acid (TFA) 5: 95: 0.1; Phase B: ACN: water: TFA 60: 40: 0.1 ) at $75{ }^{\circ} \mathrm{C}$ column temperature followed by UV detection at $215 \mathrm{~nm}$. The teriparatide 
content was determined by external calibration against the in-house RGB-10 reference standard. The effect of high temperature on the stability of the protein was tested by method validation. It was proved that it has no influence on the quality of teriparatide during analysis.

Metacresol content of the drug products was determined by UV spectrophotometry. The samples were diluted to 75 -fold with water prior to the measurement and quantitated against external standard at $272 \mathrm{~nm}$ in a $10-\mathrm{mm}$ cuvette using a Shimadzu 1600 spectrophotometer.

\subsection{Identity and Structure}

Intact molecular mass determination was carried out on the samples in their original concentrations using a Shimadzu Nexera LC-30AD liquid chromatography system coupled online to a Bruker micrOTOF-Q II high-resolution mass spectrometer. The protein was analysed by RP-HPLC using a gradient elution program. The molecular mass of teriparatide in RGB-10 and the RMP was determined by high-resolution mass spectrometry with electrospray ionisation after separation by means of liquid chromatography (LC/ESI-HRMS). The monoisotopic molecular masses were obtained through the deconvolution of the raw mass spectra. The mass accuracy of the measurement, that is, the difference between the measured and the calculated (theoretical) monoisotopic molecular masses, supplied by the instrument applied, is approximately within a range of $20 \mathrm{ppm}$.

The primary structure of teriparatide in RGB-10 and the RMP was confirmed by comparative peptide mapping [6]. Peptide fragments were analysed by liquid chromatographytandem mass spectrometry (LC-MS/MS) and their structure was identified based on their accurate molecular masses and high-resolution MS/MS spectra. This approach provided full coverage of the amino acid sequence and verified identity to the theoretical amino acid sequence of teriparatide. The primary sequences of RGB-10 and the RMP were compared with peptide mapping where Glu-C protease (Sigma, St. Louis, MO, US) was used for enzymatic digestion. The samples were injected directly into the LC-MS/MS system. The Glu-C digestion was analysed by RP-HPLC using a gradient elution programme. The MS/MS spectra were recorded according to Data Dependent Acquisition.

The higher order structure (HOS) of PTH(1-34) in RGB10 and the RMP was compared by circular dichroism (CD) spectroscopy [7-9]. The secondary structure of teriparatide was characterised by $C D$ spectroscopy in the far-UV spectral region (195-260 nm). CD spectra of the samples were recorded in two matrices, phosphate-buffered saline (PBS) $(\mathrm{pH}=7.4)$ and in PBS-trifluoroethanol (TFE) 1:1 mixture

Table 1 Overview of the critical quality attributes and the corresponding analytical methods applied

\begin{tabular}{|c|c|c|c|}
\hline Critical quality attributes & Risk of difference & Effect & Applied method \\
\hline \multicolumn{4}{|l|}{ Composition and strength } \\
\hline Protein content & Dosage accuracy & Efficacy & RP-HPLC \\
\hline Preservative content (metacresol) & $\begin{array}{l}\text { Multidose product, sterility is not main- } \\
\text { tained }\end{array}$ & Safety & Spectrophotometry \\
\hline \multicolumn{4}{|l|}{ Identity, structure } \\
\hline Intact molecular mass & Altered or lost biological activity & Safety, efficacy & ESI-HRMS \\
\hline Amino acid sequence & & & UV- and MS-based peptide mapping \\
\hline Secondary structure & & & $\mathrm{CD}$ \\
\hline Primary and higher order structure & & & 2D-NMR \\
\hline \multicolumn{4}{|l|}{ Purity, variants } \\
\hline Related compounds & Altered or lost biological activity & Safety, efficacy & $\begin{array}{l}\text { IEX-HPLC } \\
\text { RP-HPLC }\end{array}$ \\
\hline HMWs & Potential unexpected immune response & Safety, efficacy & $\begin{array}{l}\text { SE-HPLC } \\
\text { SDS-PAGE }\end{array}$ \\
\hline \multicolumn{4}{|l|}{ Biological activity and receptor binding } \\
\hline $\begin{array}{l}\text { Binding affinity, ability to activate the } \\
\text { (PTH-R1) }\end{array}$ & Altered or lost biological activity & Efficacy & $\begin{array}{l}\text { UMR-106 cell-based bioassay } \\
\text { Saos- } 2 \text { cell-based bioassay } \\
\text { Receptor binding assay }\end{array}$ \\
\hline Immunological reactivity & Potential unexpected immune response & Safety & ELISA assay \\
\hline
\end{tabular}

$C D$ circular dichroism, ELISA enzyme-linked immunosorbent assay, ESI-HRMS high-resolution mass spectrometry with electrospray ionisation, $H M W s$ higher molecular weight species, IEX-HPLC ion-exchange high-performance liquid chromatography, $M S$ mass spectrometry, $N M R$ nuclear magnetic resonance spectroscopy, $P T H-R 1$ parathyroid hormone receptor, $R P-H P L C$ reversed-phase high-performance liquid chromatography, SDS-PAGE sodium dodecyl sulphate polyacrylamide gel electrophoresis, $S E-H P L C$ size-exclusion high-performance liquid chromatography, $U V$ ultraviolet 
after buffer exchange. The recorded spectra were deconvoluted by CDNN software to calculate the ratio of the secondary structural elements.

To compare the HOS of the samples, 2D natural-abundance SoFASt ${ }^{1} \mathrm{H}_{-}{ }^{15} \mathrm{~N}$-HMQC (heteronuclear multiple quantum coherence) nuclear magnetic resonance (NMR) spectra were collected on an $800 \mathrm{MHz}$ Varian NMR system equipped with a cryoprobe (Ni: 64, TD: 1024). For each sample, $\mathrm{D}_{2} \mathrm{O}$ was used (5\%) as the lock substance. DPFGSE (double-pulsed-field-gradient-spin-echo) pulse scheme was applied for the suppression of the intensive water signal. The spectra were evaluated by visual comparison.

\subsection{Purity, Variants}

RP-HPLC experiments, using C18 column $(100 \times 2.1 \mathrm{~mm}$, S-1.7 $\mu \mathrm{m}$ ) with gradient elution (Phases A: ACN: water: TFA 5: 95: 0.1; Phase B: ACN: water: TFA 60: 40: 0.1), were performed to characterise the impurity profile. The separation was run on a Shimadzu Nexera LC-30AD UHPLC system at $60{ }^{\circ} \mathrm{C}$ column temperature, with $0.5 \mathrm{~mL} / \mathrm{min}$ flow rate for $33 \mathrm{~min}$. Samples of $10 \mu \mathrm{L}$ were injected without dilution. The peaks were detected by UV detector at $215 \mathrm{~nm}$.

Ion-exchange high-performance liquid chromatography (IEX-HPLC) analyses were performed to quantitate charge variants on an Agilent 1200 LC system equipped with a fluorescence detector. The separation was performed on a strong cation exchange column $(100 \times 4 \mathrm{~mm}, \mathrm{~S}-5 \mu \mathrm{m})$ applying salt gradient elution \{Phase A: (0.01 M sodium acetate [ $\mathrm{NaOAc}], \mathrm{pH}=5.5$ in water): ACN 55: 45; Phase $\mathrm{B}$ : (0.35 M NaOAc, $\mathrm{pH}=5.5$ in water): ACN 55: 45 . Samples of $20 \mu \mathrm{L}$ were injected and separated with a flow rate of $0.6 \mathrm{~mL} / \mathrm{min}$ and the column temperature was $25^{\circ} \mathrm{C}$. For fluorescence detection, the excitation wavelength was $280 \mathrm{~nm}$ while the detection wavelength was $360 \mathrm{~nm}$.

Size exclusion high-performance liquid chromatography (SE-HPLC) was performed using a diol column $(250 \times 4.6 \mathrm{~mm}, \mathrm{~S}-5 \mu \mathrm{m}, 6 \mathrm{~nm})$ in isocratic mode to monitor higher molecular weight species (HMWs). The composition of the mobile phase was a 75:25 mixture of $(0.05 \%$ TFA, $0.2 \mathrm{M} \mathrm{NaClO}_{4}$ in water) and acetonitrile. Samples of $8 \mu \mathrm{L}$ were injected without dilution with $0.1 \mathrm{~mL} / \mathrm{min}$ flow rate. UV detection was carried out at $215 \mathrm{~nm}$. As an orthogonal method, sodium dodecyl sulphate polyacrylamide gel electrophoresis (SDS-PAGE) under non-reducing conditions in 10-20\% Tris-tricine gel with silver staining was applied to detect the potential presence of the HMWs. Percentages of protein impurities were evaluated visually by comparing band intensities of impurities with those of the teriparatide monomer. For determination of purity by non-reducing SDS-PAGE, $1000 \mathrm{ng}, 5 \mathrm{ng}$ and $2.5 \mathrm{ng}$ (staining control) of the sample are applied on the gel, followed by electrophoretic separation. The percentages of the impurities are evaluated visually comparing the intensity of the impurity bands in the $1000 \mathrm{ng}$ application with the intensity of the $5 \mathrm{ng}$ band $(<0.5 \%, \leq 0.5 \%$ or $>0.5 \%)$. The gels were evaluated with the limit test method.

\subsection{Biological Activity and Receptor Binding}

In vivo potency assays. The potency of RGB-10 and the RMP were compared in UMR-106 rat and Saos-2 human osteosarcoma cell line-based assays. The activation of PTH-R1 (a G protein-coupled receptor) in UMR-106 and Saos- 2 cells is selectively mediated via binding of teriparatide, which leads to the release of the secondary messenger molecule cyclic adenosine monophosphate (cAMP). cAMP release is measured by enzyme fragment complementation (EFC) technique using the HitHunter cAMP XS Fluorescence Kit (DiscoverX, Fremont, CA, US).

Dilution series of the teriparatide reference standard and the investigated sample were prepared in triplicate and were incubated on a microtitre plate with starved UMR106 or Saos- 2 cells. Incubation took place in the presence of 3-isobutyl-1-methylxanthine (IBMX) while the cAMP release was measured by the EFC technique.

Relative potency (RP) of the sample was calculated relative to the in-house product-specific standard (which had been calibrated against the actual WHO standard in International Units) [10]. The samples were analysed side by side against a product-specific reference standard in parallel assays performed by the same operator.

The receptor binding activity (RBA) of the RGB-10 and the RMP samples to PTH-R1 were determined by competitive enzyme-linked immunosorbent assay (ELISA). The assay was based on the immobilisation of PTH-R1 (ECD 23-189, human recombinant protein fragment, R\&D Systems) onto streptavidin-coated plates using biotinylated PTH(1-34) (Anaspec, Fremont, CA, US). The addition of teriparatide displaces biotinylated $\mathrm{PTH}(1-34)$ from the receptor; bound receptor was detected by a primary $\mathrm{PTH}$ R1-specific antibody and by an HRP-conjugated secondary anti-sheep antibody. After concentration of the samples by use of Amicon Ultra $3 \mathrm{~K}$ centrifuge filter devices, dilution series of teriparatide reference standard and the investigated sample were prepared in triplicate. The compared samples (RGB-10 and RMP) were analysed side by side in parallel assays performed by the same operator.

Immunological reactivity of RGB-10 and the RMP was tested by a sandwich ELISA based on a commercially available homogeneous ELISA kit (High Sensitivity Human PTH(1-34) ELISA, Co. Immutopics, Immundiagnostik, Bensheim, Germany) with appropriate modifications. The test was basically a two-site enzyme-linked immunosorbent assay, which uses biotinylated affinity purified goat polyclonal antibody (pAb) for capture. 
Human PTH(1-34)-specific pAbs conjugated with horseradish peroxidase (HRP) were applied for detection.

Dilution series of teriparatide reference standard and the investigated samples were prepared in duplicates and were incubated with the antibodies in a homogeneous assay format. To test the immunological reactivity of RGB-10 and the RMP, the two samples were analysed side by side within one assay relative to the product-specific reference.

\subsection{Statistical Evaluation}

The results of the different tests were evaluated based on pre-defined comparability criteria. In the case of qualitative methods such as NMR spectroscopy or primary structure, the comparability requirement was defined as the high correspondence between the test sample and the reference by visual inspection. In the case of the quantitative tests, the relevant comparability ranges were generated by measuring several batches of the reference product. Data were evaluated by descriptive statistics in line with the relevant guidelines [3]; the age dependence of the parameters was also considered. The comparability ranges for biosimilarity assessment were established based on the measured RMP data considering batch-to-batch variability. Comparability ranges were determined as $95 \%$ prediction interval of the measured data. If data indicated a correlation between the age and the parameters of the drug product studied, as in the case of metacresol and teriparatide content, the adequate upper or lower limit of the prediction interval was taken into consideration.

\section{Results}

\subsection{Composition and Strength}

The API content of the injection was tested by RP-HPLC method. Considering the $250 \mu \mathrm{g} / \mathrm{mL}$ label claim of the RMP as $100 \%$, the API contents of the RGB-10 and RMP batches tested were found to be $102 \%$ and $99 \%$, respectively. The results were within the pre-defined comparability range; the API content of the two products were considered comparable.

The labelled concentration of added metacresol is the same, $0.3 \% \mathrm{w} / \mathrm{v}$ (defined as $100 \%$ ) in both DPs. The metacresol content was determined by UV spectrophotometry and was found to be $99 \%$ for the RMP and $100 \%$ for RGB-10. The measured data met the criterion of $90-110 \%$ defined for preservatives in EMA guideline $3 \mathrm{AQ} 11 \mathrm{a}$, so RGB-10 and the RMP were proven to be comparable in this respect as well.

\subsection{Identity and Structure}

The accurate mass measured for RGB-10 was 4115.1785 Da (11.6 ppm), while it was 4115.1566 Da (6.3 ppm) for the RMP. The theoretical monoisotopic mass of the teriparatide molecule is $4115.1309 \mathrm{Da}$ $\left(\mathrm{C}_{181} \mathrm{H}_{291} \mathrm{~N}_{55} \mathrm{O}_{51} \mathrm{~S}_{2}\right)$. The monoisotopic molecular mass of teriparatide in both RGB-10 and RMP is in good agreement with the corresponding theoretical monoisotopic mass value, considering the mass accuracy of the instrument applied.

The MS/MS spectra of the teriparatide peptide fragments in RGB-10 and the RMP were compared with each other. The retention time, the accurate molecular mass and the high-resolution MS/MS spectra of the peptide components were identical in the two DPs, proving that the primary structure of teriparatide is identical in the two products. Furthermore, this approach also verified identity to the theoretical amino acid sequence of teriparatide. In the UV chromatograms, seven identical peptide fragments (G1-G7) were identified in each teriparatide-containing sample (Fig. 1). The peak of the metacresol excipient could be observed by UV both in RGB-10 and the RMP. Based on the results of the peptide mapping, the amino acid sequences of teriparatide in RGB-10 and the RMP are identical. On the basis of the MS-based structural characterisation (intact molecular mass determination and UV- and MS-based peptide mapping analyses), the primary structures of teriparatide in the RGB-10 drug product and the RMP are in full agreement.

The appropriate HOS of proteins is a prerequisite for biological functionality. Unexpected variations in the protein structure can lead to loss of function or may trigger aggregation [11]. Although in aqueous solution teriparatide adopts a predominantly disordered structure, the $\alpha$-helix ratio increases drastically during receptor binding, while in bound form the molecule adopts $\alpha$-helical conformation [7]. The secondary structure of RGB-10 and RMP was compared by CD measurement [7-9] in PBS buffer with and without the addition of TFE. The role of the TFE is to test $\alpha$-helix-forming propensity. In both buffers, the CD spectra of investigated RGB-10 and RMP samples showed very good similarity, which is proof of the structural comparability and similar helix forming propensity. CD spectra of RGB-10 and the RMP showed high similarity in both PBS and PBS: TFE buffers (Fig. 2). The calculated $\alpha$-helix ratio in PBS $(\mathrm{pH}=7.4)$ was found to be $24.4 \%$ and $25.2 \%$ for the RMP and RGB-10, respectively, and increased to $85.1 \%$ and $84.3 \%$, respectively, in the PBS:TFE mixture. The calculated $\alpha$-helix ratios are consistent with data from literature [12] for the various media; that is, $24-28 \%$ for $\mathrm{pH} \sim 7$ and $70 \%$ or higher in the presence of $50 \%$ TFE. The difference is $<1 \%$ in both cases, so the results are considered equivalent for the two drug 


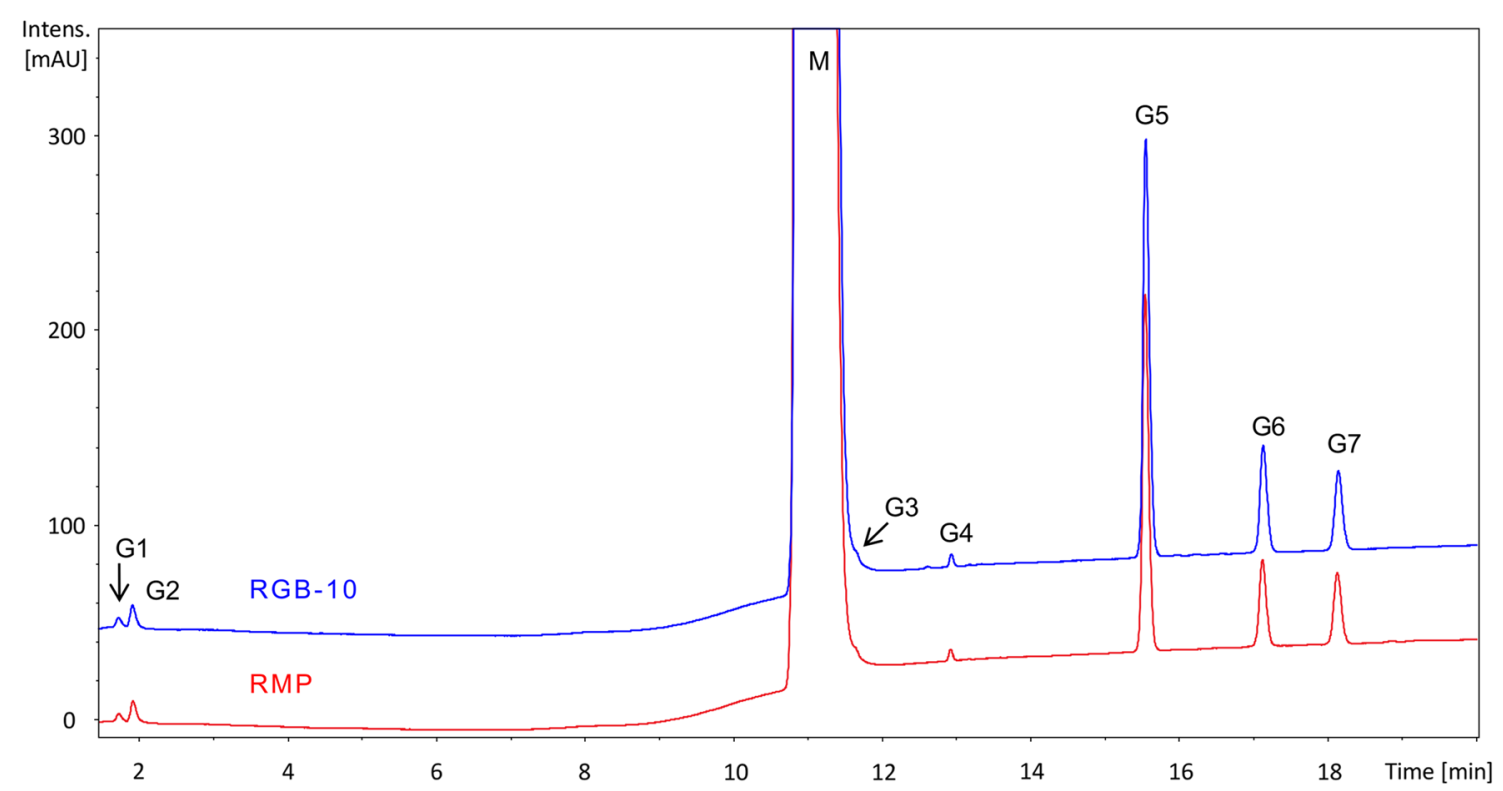

Fig. 1 UV peptide mapping chromatograms $(215 \mathrm{~nm})$ of the Glu-C digested samples of RGB-10 and RMP drug products. Major components: G1: 20-22, G2: 1-4, G3: 31-34, G4: 23-30, G5: 23-34, G6:

products substantiating highly similar structural propensities of recombinant teriparatide in the RMP and RGB-10.

The 2D SoFASt ${ }^{1} \mathrm{H}^{-15} \mathrm{~N}-\mathrm{HMQC}$ spectra of both RGB-10 and the RMP were compared and evaluated visually (Fig. 3). In the 2D NMR spectra, 33 peaks belonging to the backbone amide groups were detected, while other peaks belong to amide groups situated in side chains of asparagine, glutamine, lysine and tryptophan residues. The complete overlap of the peaks in the two spectra clearly demonstrates the

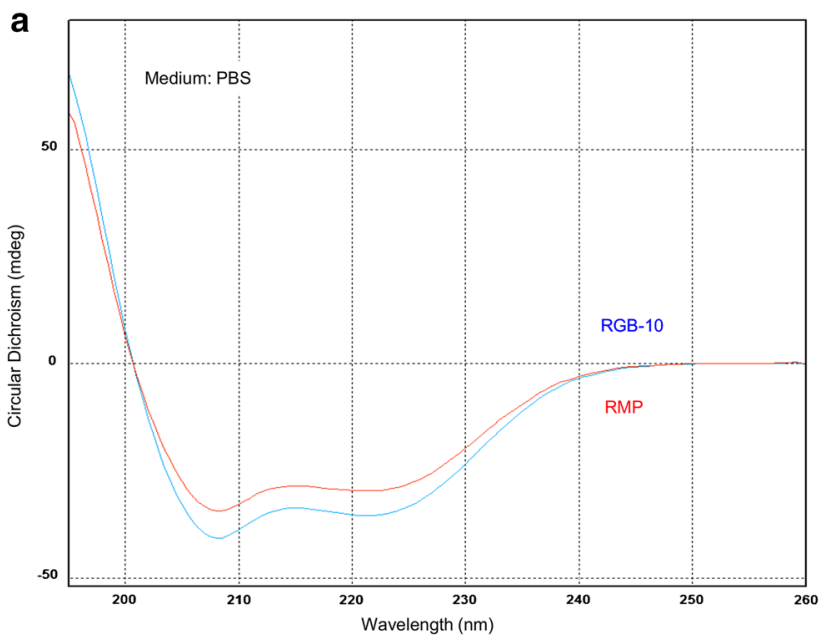

5-19, G7: 5-22 peptide fragments. $M$ metacresol, $R M P$ reference medicinal product

structural similarity of the two drug substances at the atomic level.

\subsection{Purity, Variants}

The purity of RGB-10 and RMP was determined by RPHPLC (hydrophobicity based) and IEX-HPLC (charge based) methods to cover each potential degradation product, in line with the CQAs. Age dependence of the amount of the degradation products was observed by both chromatographic

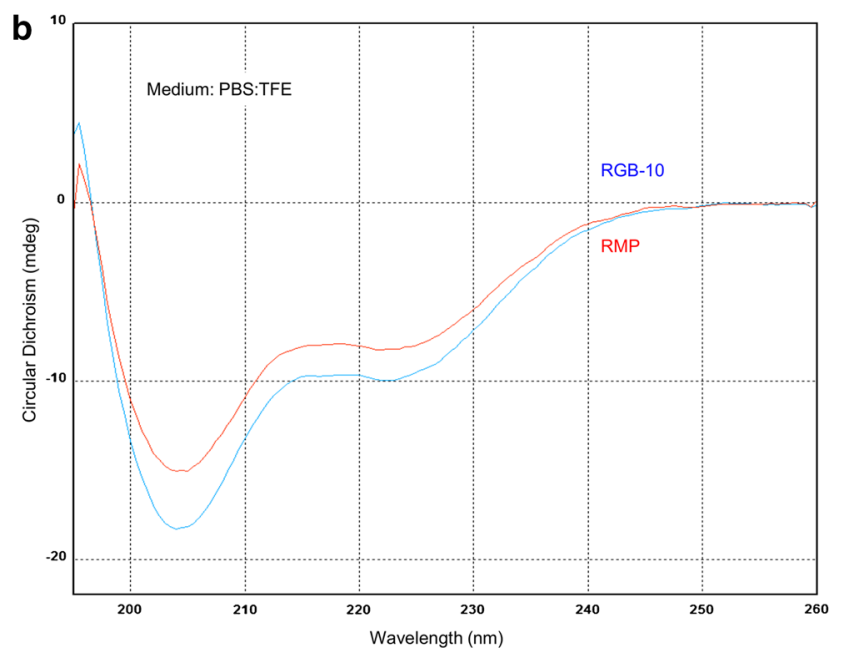

Fig. 2 CD spectra of RGB-10 and the RMP in different media: a in PBS, $\mathbf{b}$ in PBS-TFE (1:1). $C D$ circular dichroism, $P B S$ phosphate-buffered saline, $R M P$ reference medicinal product, TFE trifluoroethanol 
methods. Stability and stress studies demonstrated that the degradation products were the same with similar degradation kinetics (data not shown). A similar age-dependent degradation profile of teriparatide was observed in both RMP and RGB-10 in stability studies. Therefore, differences in the age of the tested samples resulted in a difference in their HPLC chromatograms. RP-HPLC profiles of RGB-10 and the RMP are shown in Fig. 4. The overall chromatographic pattern is shown to be similar. The only two minor differences observed at 17.0 and 17.9 min were identified as agedependent degradation products, which are characteristic impurities of the RMP.

IEX-HPLC results exhibited a coherent picture on the quality of RGB-10 and the RMP; that is, highly similar patterns with differences related to either age or a productspecific deviation (Fig. 5). An impurity characteristic to the RMP was detected at 54.0 min.

The presence of HMWs was monitored using SE-HPLC and SDS-PAGE as orthogonal methods. Based on the data obtained for the determination of the comparability range, no HMWs were detected by SE-HPLC in RGB-10 and the RMP, not even at the detection limit of the method $(0.05 \%)$. The head-to-head comparability study showed no aggregates were detected by SE-HPLC. Moreover, no other band than that of teriparatide monomer was detected in the RGB-10 and RMP batches tested by non-reducing SDS-PAGE (data not presented). This result corroborated the data from the SE-HPLC analysis and is in line with the literature reporting a low aggregation tendency of teriparatide [7]. In conclusion, the aggregation propensity of the compared DPs is proven to be identical and fulfils the requirement of comparability.

\subsection{Biological Activity and Receptor Binding}

In the routine potency assay for teriparatide, cAMP release is determined by use of rat osteosarcoma cell line UMR-106, which is also used in six of seven laboratories participating in the collaborative study for characterisation of first International human recombinant $\mathrm{PTH}(1-34)$ WHO standard (NIBSC code 04/200) [10]. The potency of RGB-10 and RMP were compared in two cell-based assays (cAMP-release assay using a rat and a human osteosarcoma cell line, UMR-106 and Saos-2, respectively) and an ELISA-based receptor-binding assay. The UMR-106 and Saos-2 assays are not orthogonal methods by their molecular mechanisms but they use cell lines from different species. Nevertheless, as a rat cell line is used as routine release and stability assay, additional comparability data were generated with the human cell line as supportive data.

In both assays, products were analysed side by side against a product-specific reference standard and the results

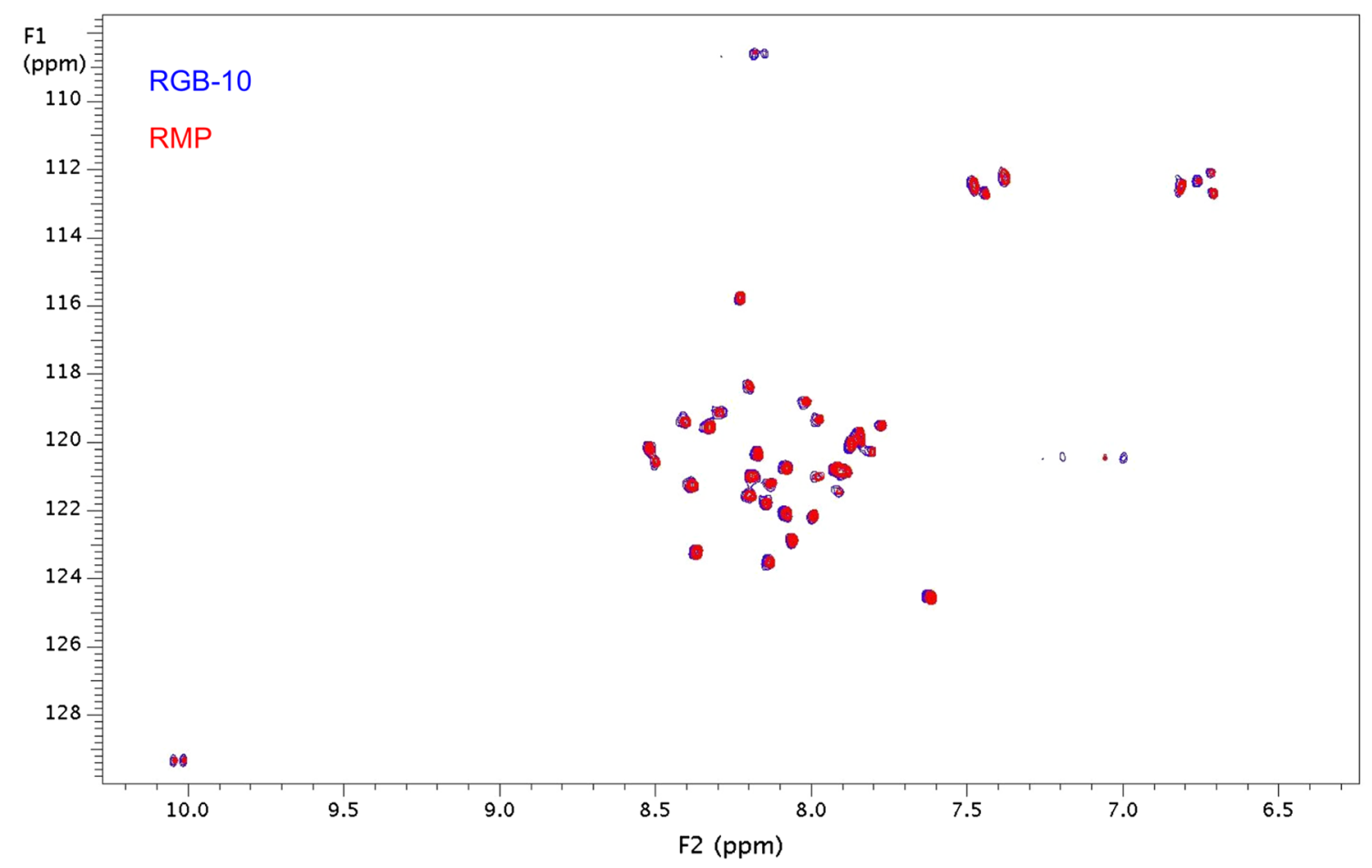

Fig. 3 The $2 \mathrm{D}{ }^{1} \mathrm{H}_{-}{ }^{15} \mathrm{~N}$ HMQC NMR spectra of RGB-10 and RMP show high level of similarity of the primary and the higher order structures of the DPs. DPs drug products, $H M Q C$ heteronuclear mul- tiple quantum coherence, $N M R$ nuclear magnetic resonance spectroscopy, $R M P$ reference medicinal product 


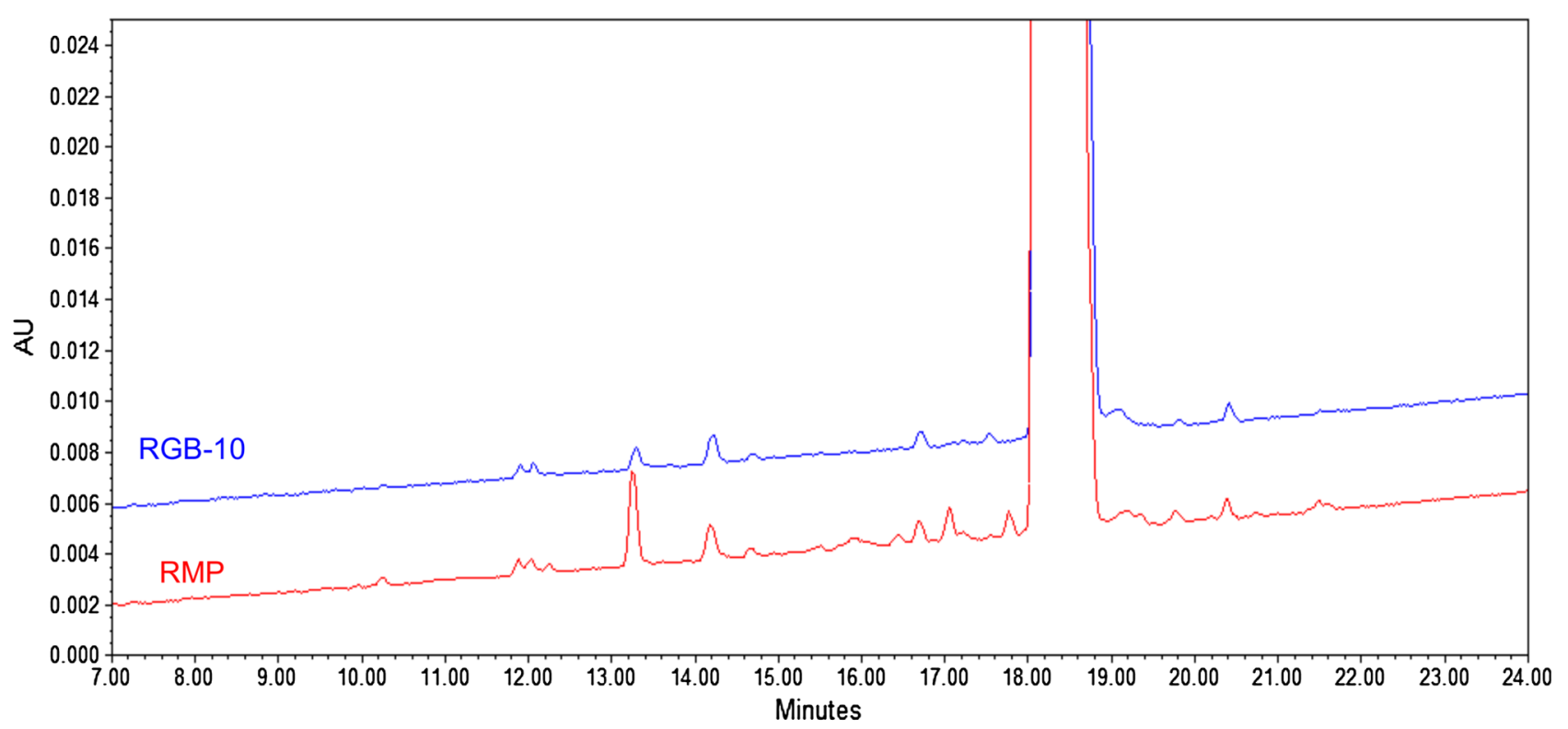

Fig. 4 Reversed-phase high-performance liquid chromatography (RP-HPLC) chromatograms of RGB-10 and the reference medicinal product (RMP)

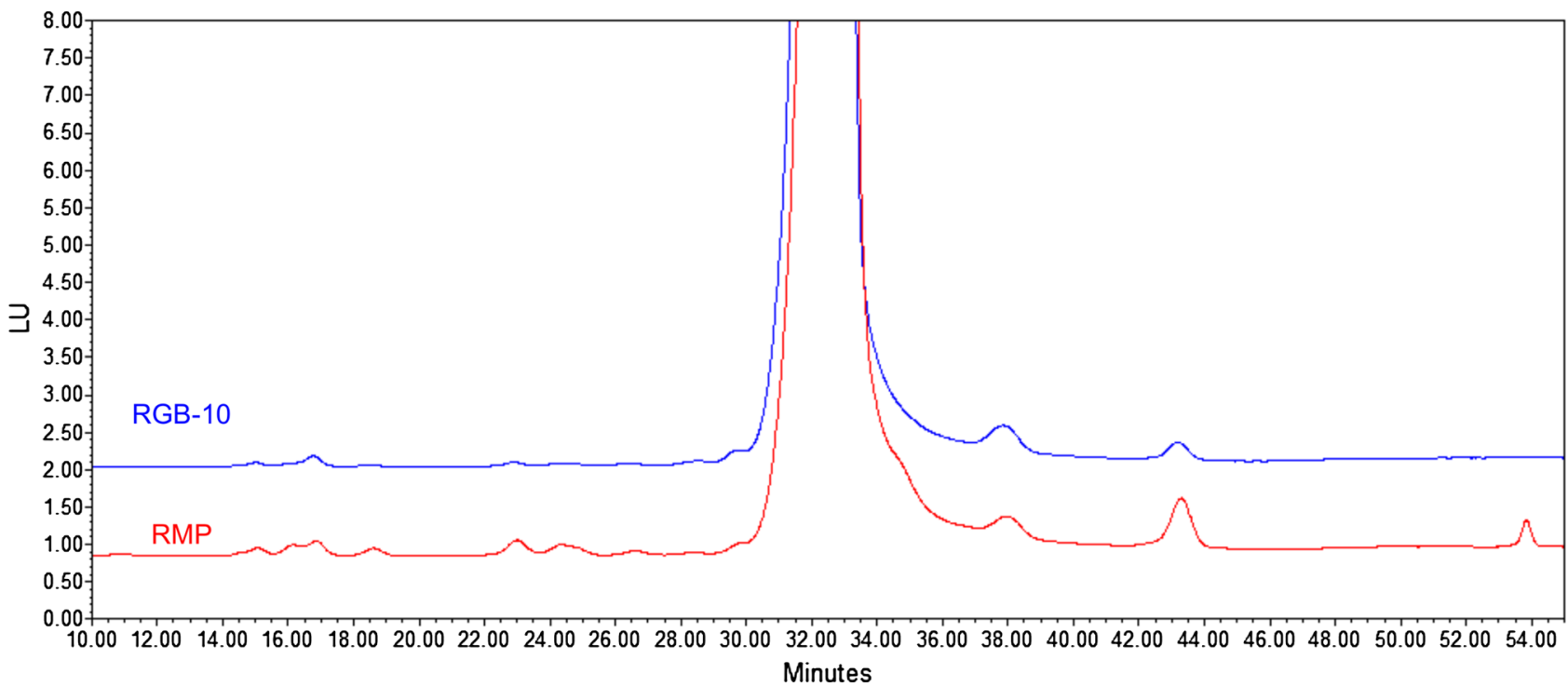

Fig. 5 Ion-exchange high-performance liquid chromatography (IEX-HPLC) chromatograms of RGB-10 and the reference medicinal product (RMP)

were reported as a geometric mean of three independent assays. Representative results (dose-response curves) from side-by-side analysis in each assay are presented in Figs. 6, 7 , and 8 .

Immunologic reactivity was tested by competitive ELISA. The products were analysed side by side within one assay against a product-specific reference standard in three independent assays and the potency for each product was calculated as a combined result of the three assays. Representative results (dose-response curves) from side-by-side analysis in one assay are presented in Fig. 9.

Side-by-side analyses of several batches of the two products with regard to potency and immunologic reactivity (each in the three independent assays) demonstrated great similarity between RGB-10 and the RMP (Table 2). Comparison of the dose-response curves showed similar 
reactivity of the two products regarding cAMP-release reaction, PTH-R1 receptor binding and immunological reactivity. The final (combined) potency results were within the pre-defined comparability range for each assay, so the two products were found to be comparable.

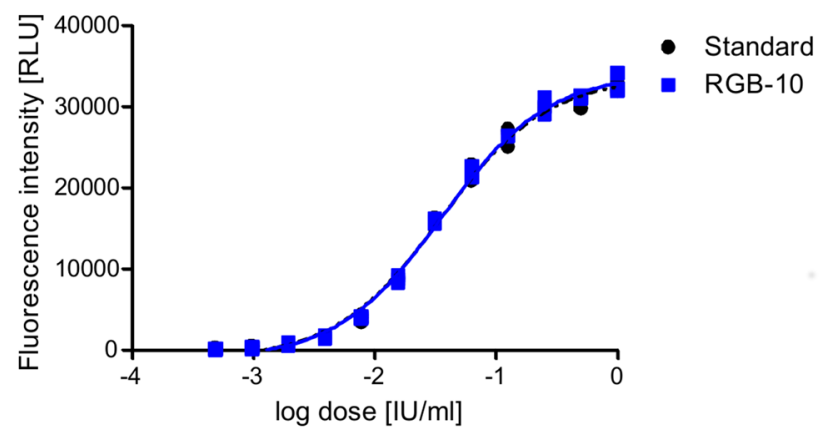

\section{Discussion}

Results from the extensive comparative physicochemical and biological characterisation, using a wide range of stateof-the-art, orthogonal methods, prove similarity of RGB-10 to its reference medicinal product at the quality level. The quality comparability study was designed to investigate all the critical quality attributes covering all the relevant features of the protein necessary for appropriate functionality. Identity tests entailed analysis of the content of active

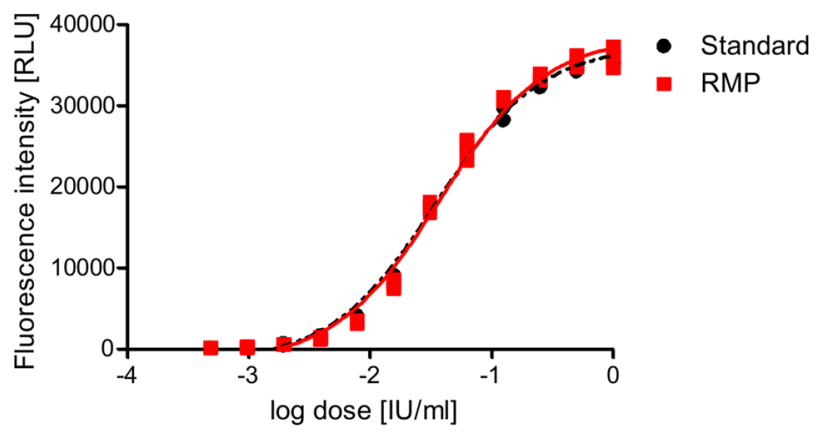

Fig. 6 Representative figure (dose-response curves) of side-by-side cAMP-release assay (UMR-106 cells) of RGB-10 (blue, left graph) and reference medicinal product (RMP) (red, right graph) analysed relative to reference standard (black)
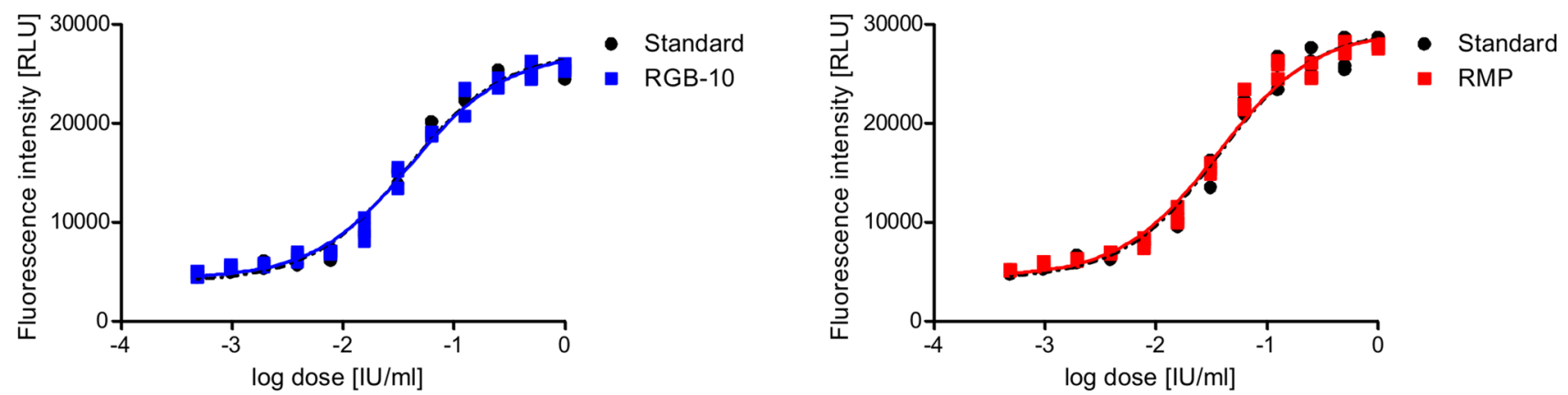

Fig. 7 Representative figure (dose-response curves) of side-by-side cAMP-release assay (Saos-2 cells) of RGB-10 (blue, left graph) and reference medicinal product (RMP) (red, right graph) analysed relative to reference standard (black)
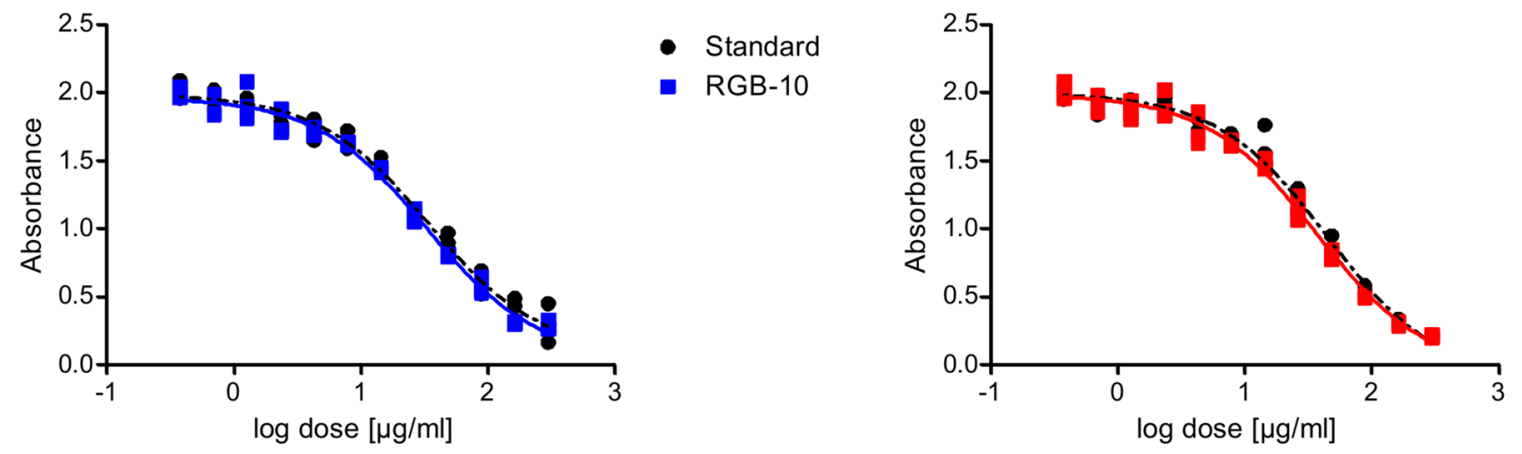

- Standard

- Standard

- RGB-10

Fig. 8 Representative figure (dose-response curves) of side-by-side receptor-binding assay of RGB-10 (blue, left graph) and reference medicinal product (RMP) (red, right graph) analysed relative to reference standard (black) 


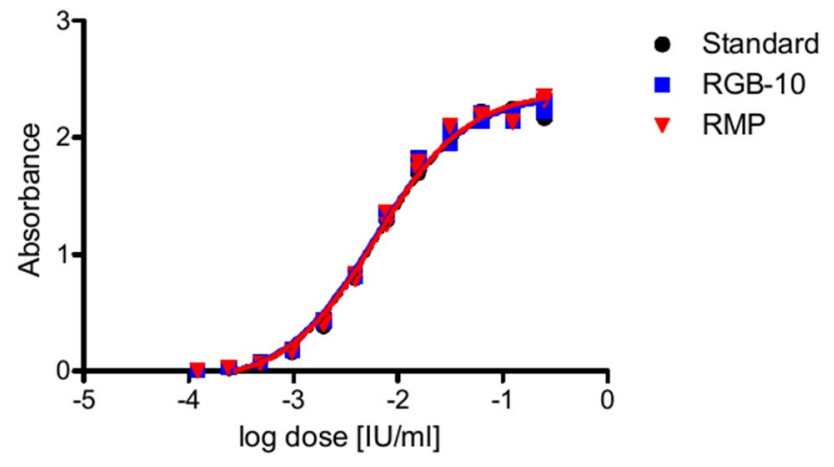

Fig. 9 Representative figure (dose-response curves) of side-by-side enzyme-linked immunosorbent assay (ELISA) of reference medicinal product (RMP) (red) and RGB-10 (blue) analysed relative to reference standard (black) to reveal specific immunologic reactivity

ingredients and the excipients, the primary and the higher order molecular structure, purity in respect to the proteinrelated compounds and the high molecular weight species as well as the biological activity.

The API content has a significant impact on the efficacy of the drug product as it determines the dose accuracy, while a precise amount of the preservative agent (metacresol) is necessary to maintain sterility. The measurements reveal identical concentrations of the API and metacresol in both DPs.

As a general requirement, the API of a biosimilar candidate must have an identical amino acid sequence to the reference medicinal product [3]. The amino acid sequence of proteins can be compared and determined by peptide mapping through proteolytic cleavage of the polypeptides followed by comparison of the HPLC-UV chromatograms of the digestion and the MS-based identification of the specific peptide components. Besides the determination of the position and type of the amino acid residues in a polypeptide chain, this approach is sensitive to post-translational and chemical modifications, like oxidation or deamidation. The HPLC-UV and MS total ion chromatogram peptide map chromatograms of the tryptic peptides of biosimilar RGB-10 and RMP are technically indistinguishable, suggesting $100 \%$ sequence identity. This result, together with the results of
ESI-MS intact molecular mass measurements confirms the identity of the primary structure of RGB-10 and RMP samples. The natural abundance of ${ }^{15} \mathrm{~N}-{ }^{1} \mathrm{H}$ HMQC NMR pulse sequences proved to be the analytical tools particularly suitable for the investigation of the HOS of therapeutic protein drug substances [13]. In the $2 \mathrm{D}{ }^{15} \mathrm{~N}-{ }^{1} \mathrm{H}$ heteronuclear multiple quantum coherence (HMQC) spectrum, each peak is linked directly to an NH moiety while their shape and position heavily depend on the chemical environment of the corresponding functional group. $\alpha-\mathrm{NH}$ groups of the proteins are constituents of the protein backbone, consequently $2 \mathrm{D}$ ${ }^{15} \mathrm{~N}-{ }^{1} \mathrm{H} \mathrm{HMQC}$ spectra are sensitive fingerprint-like indicators of the changes in the primary and the HOSs. The results of 2D NMR measurements show structural identity between RGB-10 and RMP with respect to constitution, configuration and conformation.

Teriparatide is highly sensitive to the various degradation processes, like oxidation or deamidation [14-16], which may influence the binding affinity and the biological activity [17]. Similar impurity profile of RGB-10 and RMP was observed with chromatographic methods that included RP- and IEXHPLC. Minor differences were found in the samples with different age; however, these differences had no influence on biological activity. Overall, the results from size-exclusion chromatography (SE-HPLC) and SDS-PAGE shows that HMWs are not detected either in the RGB-10 or in the RMP samples.

Comprehensive sets of UMR-106 and Saos2 cell-based potency bioassays and ELISA-based receptor-binding assays were developed to evaluate and compare the functional integrity of RGB-10 and the RMP. As the detection of late responses (e.g. effects on bone growth) cannot be measured in vitro, the focus was on the measurement of the binding of PTH(1-34), induction of the second messenger cAMP (in murine as well as in human cell-line expression PTH-R1), and detection of immunologic reactivity.

The potency of the RGB-10 and the RMP drug products was compared using the PTH-R1 binding receptor assay, and the side-by-side analysis of several batches of the two DPs demonstrate high similarity with regard to potency and immunologic reactivity (each in three independent assays). Comparison of the dose-response curves and relative

Table 2 Results of biological characterisation

\begin{tabular}{lllll}
\hline Parameter & Method & $\begin{array}{l}\text { Comparability range } \\
\text { (IU/mg) }\end{array}$ & RGB-10 DP (IU/mg) & Evaluation \\
\hline Biological activity & Cell-based bioassay, UMR-106 cells & $7770.1-11,622$ & $9325.0-10,458.6$ & Comparable \\
& Cell-based bioassay, Saos-2 cells & $8232.7-13,132.2$ & $9116.5-12,223.4$ & Comparable \\
& Receptor-binding assay & $89.49-130.41$ & $104.14-107.16$ & Comparable \\
Immunologic reactivity & ELISA & $9085.6-12,332.4$ & $9635.7-11,312.4$ & Comparable \\
\hline
\end{tabular}

ELISA enzyme-linked immunosorbent assay 
potency values showed similar reactivity of the two products regarding cAMP-release reaction, PTH-R1 receptor binding and immunological reactivity. The final (combined) potency results were within the preliminary defined comparability range for each assay. Therefore, the two products were considered to be comparable. The extensive characterisation of the predefined CQAs demonstrated a high degree of similarity between RGB-10 and the RMP.

\section{Conclusion}

The results of the present quality comparability exercise formed the basis for the specifically designed clinical programme entailing a single pharmacokinetic/pharmacodynamic study, which demonstrated bioequivalence, and supported the approval of the marketing authorisation application of RGB-10 in the European Union.

Acknowledgements Ivett Szkiba-Kovács and Dániel Hüse are acknowledged for their participation in the measurements and evaluation; Katalin Baranyáné Ganzler, Rita Ocskay and Csaba Szántay Jr. for their contribution to compilation of the study; and Enikő Jókai for medical and linguistic review.

\section{Compliance with Ethical Standards}

Funding No external funding was received for the conduct of this study.

Conflict of interest Piroska Kovács, Tamás Schäfer, Viktor Háda, Helga Hevér, Sonja Klingelhöfer, Merle Nebel, Tanja Stadie, Róbert Kiss, and Zoltán Urbányi acknowledge potential conflicts of interest as current employees of Gedeon Richter Plc. or Richter Helm Biologics GMBH \& Co KG.

Open Access This article is distributed under the terms of the Creative Commons Attribution-NonCommercial 4.0 International License (http://creativecommons.org/licenses/by-nc/4.0/), which permits any noncommercial use, distribution, and reproduction in any medium, provided you give appropriate credit to the original author(s) and the source, provide a link to the Creative Commons license, and indicate if changes were made.

\section{References}

1. Quattrocchi E, Kourlas H. Teriparatide: a review. Clin Ther. 2004;26:841-54.
2. Bodenner D, Redman C, Riggs A. Teriparatide in the management of osteoporosis. Clin Interv Aging. 2007;2:499-507.

3. European Medicines Agency. Guideline on similar biological medicinal products 2014 CHMP/437/04 Rev 1.

4. Takács I, Jókai E, Kováts DE, Aradi I. The first biosimilar approved for the treatment of osteoporosis: results of a comparative pharmacokinetic/pharmacodynamic study. Osteoporos Int. 2019;30:675-83.

5. International Conference on Harmonization. Pharmaceutical development Q8(R2). 2009.

6. Háda V, Bagdi A, Bihari ZS, Timári SB, Fizil Á, Szántay CS. Recent advancements, challenges, and practical considerations in the mass spectrometry-based analytics of protein biotherapeutics: a viewpoint from the biosimilar industry. J Pharm Biomed Anal. 2018;161:214-38.

7. Jin L, Briggs SL, Chandrasekhar S, Chirgadze NY, Clawson DK, Schevitz RW, Smiley DL, Tashjian AH, Zhang F. Crystal structure of human parathyroid hormone 1-34 at 0.9-A resolution J Biol Chem. 2000;275:27238-44.

8. Cohen FE, Strewler GJ, Bradley MS, Carlquist M, Nilsson M, Ericsson M, Ciardelli TL, Nissenson RA. Analogues of parathyroid hormone modified at positions 3 and 6 . Effects on receptor binding and activation of adenylyl cyclase in kidney and bone. $\mathrm{J}$. Biol. Chem. 1991;266:1997-2004.

9. Wray V, Federau T, Gronwald W, Mayer H, Schomburg D, Tegge W, Wingender E. The Structure Of Human Parathyroid Hormone From a Study of Fragments in Solution Using 1H NMR Spectroscopy and Its Biological Implications Biochemistry. 1994;33:1684-93.

10. Rafferty B, Gaines-Das RE. WHO international collaborative study of the proposed 1st international standard for parathyroid hormone 1-34, recombinant, human. 2007. WHO/BS/07.2063.

11. Philo JS, Arakawa T. Mechanisms of protein aggregation. Curr Pharm Biotechnol. 2009;10:348-51.

12. Strickland LA, Bozzato RP, Kronis KA. Structure of human parathyroid hormone(1-34) in the presence of solvents and micelles. Biochemistry. 1993;32:6050-7.

13. Kiss R, Fizil Á, Szántay CS. What NMR can do in the biopharmaceutical industry. J Pharm Biomed Anal. 2018;147:367-77.

14. Nabuchi Y, Fujiwara E, Ueno K, Kuboniwa H, Asoh Y, Ushio $\mathrm{H}$. Oxidation of recombinant human parathyroid hormone: effect of oxidized position on the biological activity. Pharm Res. 1995;12:2049-52.

15. Nabuchi Y, Fujiwara E, Kuboniwa H, Asoh Y, Ushio H. The stability and degradation pathway of recombinant human parathyroid hormone: deamidation of asparaginyl residue and peptide bond cleavage at aspartyl and asparaginyl residues. Pharm Res. 1997; 14:1685-90.

16. Kothari R, Kumar V, Jena R, Tunga R, Tunga BS. Modes of degradation and impurity characterization in rhpth (1-34) during stability studies. PDA J Pharm Sci Technol. 2011;65:348-62.

17. Rosenblatt M. Parathyroid hormone: chemistry and structureactivity relations. Pathobiol Annu. 1981;11:53-86. 